\title{
Reduced-order Model-based Feedback Controller Design for Subsonic Cavity Flows
}

\author{
X. Yuan ${ }^{1}$, E. Caraballo ${ }^{2}$, P. Yan ${ }^{1}$, H. Özbay ${ }^{1, \dagger}$, A. Serrani ${ }^{1}$, \\ J. DeBonis ${ }^{3}$, J. H. Myatt ${ }^{4}$, M. Samimy ${ }^{2, *}$ \\ Collaborative Center of Control Science \\ The Ohio State University \\ ${ }^{1}$ Department of Electrical and Computer Engineering \\ 2 Department of Mechanical Engineering \\ $\dagger$ Currently on leave at Bilkent Univ., Dept. of Electrical and Electronics Eng., Ankara, Turkey \\ 3 NASA Glenn's Quiet Aircraft Technology Program \\ 4 Air Force Research Laboratory, Air Vehicles Directorate, WPAFB \\ * Corresponding author: samimy.1@osu.edu, 614-292-6988
}

This paper explores feedback controller design for cavity flows based on reduced-order models derived using Proper Orthogonal Decomposition (POD) along with Galerkin projection method. Our preliminary analysis shows that the equilibrium of the POD model is unstable and a static output feedback controller cannot stabilize it. We develop Linear Quadratic (LQ) optimal state feedback controllers and LQ optimal observers for the linearized models. The linear controllers and observers are applied to the nonlinear system using simulations. The controller robustness is numerically tested with respect to different POD models generated at different forcing frequencies. An estimation for the region of attraction of the linear controllers is also provided.

\section{Introduction}

The flow control group within the Collaborative Center of Control Science at The Ohio State University has been working on a collaborative approach towards the design of reduced-order model-based feedback control of cavity flows. ${ }^{1,2}$ The components include obtaining detailed data using numerical simulation of or experimental work in the cavity flow, derivation of reduced-order model of the flow dynamics, ${ }^{3}$ controller design, ${ }^{4,5}$ and experimental implementation of the controller. ${ }^{6,7}$ Cavity flow control problem has received significant attention in recent years. ${ }^{8,9}$ Particularly, closed-loop control approaches have begun to gain popularity within the fluid dynamics community. ${ }^{10-14}$ From the system-theory point of view, the most outstanding difficulty in approaching cavity flow control comes from the nature of the governing NavierStokes equations, resulting in an intractable nonlinear infinite dimensional system. Physical-based linear models ${ }^{11}$ have provided a certain degree of physical insight, useful for controller design and analysis. However, linear controllers designed from these classes of models have met limited success in experiment. ${ }^{5}$ Typically, controllers designed based on linear models are capable of suppressing an oscillation mode in the cavity, but lead to the generation of other modes. It is fair to say that in the case of cavity flows, linear models may not be that useful to be employed in the design of feedback controller. It is also clear that the derivation of

Copyright (c) 2005 by the American Institute of Aeronautics and Astronautics, Inc. The U.S. Government has a royalty-free license to exercise all rights under the copyright claimed herein for Governmental purposes. All other rights are reserved by the copyright owner. 
a low-order, finite-dimensional model of the cavity flow dynamics is essential in any successful development of a feedback control strategy.

In recent years, Proper Orthogonal Decomposition (POD) along with Galerkin projection method has been increasingly used in many flow problems. ${ }^{15-18}$ By means of POD and Galerkin projection method, a system described by partial differential equations can be reduced to a finite set of ordinary differential equations. By a boundary control separation method, it is possible to incorporate the control input explicitly in the reduced order model, which is desirable from the point of view of the control design. Feedback control techniques seem to offer a considerable advantage over approaches based on open-loop forcing in terms of robustness; however, the effects of the control on the flow dynamics in closed-loop are not well understood yet. As a result, the systematic design of feedback controllers for cavity flows is still a widely open problem.

In this paper, we focus present preliminary results on feedback control design using POD-based models for cavity flows. Starting with basic equilibrium analysis of forced low dimensional models, we consider the role of linear static output feedback controller and linear-quadratic optimal state feedback controller/observer. The goal is to gain insight into the intrinsic limitations of linear feedback methodologies when applied to the specific nonlinear POD model, in particular with respect to the achievable regions of attraction and the robustness under parametric uncertainties.

This paper is organized as follows: a description of the POD based reduced-order model is given in Section II. Equilibrium analysis and static output feedback controller design are presented and discussed in Section III. In Section IV, optimal LQ controllers and observers are presented. Simulations are given in Section V, where robustness is investigated, and the regions of the attraction are estimated numerically. Finally concluding remarks are given in Section VI.

\section{Reduced Order POD Modeling}

The Navier-Stokes equations governing the flow dynamics are a set of highly nonlinear partial differential equations which are not suitable for feedback controller design. To obtain a low dimensional model of the flow where the control input appears explicitly, ${ }^{19}$ Proper Orthogonal Decomposition (POD), Galerkin projection as well as a control separation method ${ }^{20}$ were adopted. The nonlinear POD based model of the flow dynamics used in this paper is the one derived in Samimy et al, ${ }^{2}$ which reads as

$$
\dot{a}^{k}(t)=b^{k}+\sum_{j=1}^{N} d^{j k} a^{j}(t)+\sum_{j=1}^{N} \sum_{m=1}^{N} g^{j m k} a^{j}(t) a^{m}(t)+\left\langle e^{k}, \Gamma(t)\right\rangle+\sum_{j=1}^{N}\left\langle f^{j k}, \Gamma(t)\right\rangle a^{j}(t), \quad 1 \leq k \leq N,
$$

being $a^{i}(t)$ the time coefficient associated with the $i$-th POD mode. The constant scalar coefficients $b^{k}, d^{j k}$, $g^{j m k}$, and the constant vectors $e^{k}$ and $f^{j k}$ are obtained from Galerkin projection, while

$$
\Gamma(t)=\left[\begin{array}{c}
\Gamma_{u}(t) \\
\Gamma_{v}(t) \\
\Gamma_{c}(t)
\end{array}\right]
$$

is the forcing input with components $\Gamma_{u}(t), \Gamma_{v}(t)$ and $\Gamma_{c}(t)$ defined by

$$
\begin{aligned}
& \Gamma_{u}(t)=V(t) \cos (\alpha) \\
& \Gamma_{v}(t)=\left\{\begin{array}{ll}
V(t) \sin (\alpha) & V(t)>0 \\
0 & V(t) \leq 0
\end{array}\right\} \\
& \Gamma_{c}(t) \approx 1,
\end{aligned}
$$

where $\alpha=\pi / 6$. The reader is referred to Samimy et $a l^{2}$ for details. The coefficients of the POD model have been derived from CFD simulations conducted in absence of external input $(V(t)=0$, i.e., the baseline case) and in presence of an external sinusoidal excitation of the form $V(t)=A \sin \left(2 \pi f_{c} t\right)$, with $f_{c}=500 \mathrm{~Hz}$ and $f_{c}=900 \mathrm{~Hz}$ respectively. The nonsmooth nonlinearity in $\Gamma_{v}(t)$ results from the numerical computations. For the purpose of simplifying the subsequent analysis, we approximate $\Gamma_{v}(t)$ by $\Gamma_{v}(t)=V(t) \sin (\alpha)$. Simulation results show that this simplification has no significant impact on the output pressure, as seen in Figure 1 , which compares simulations of the output pressure for the forced cavity flow at Mach 0.38. Also, note that 


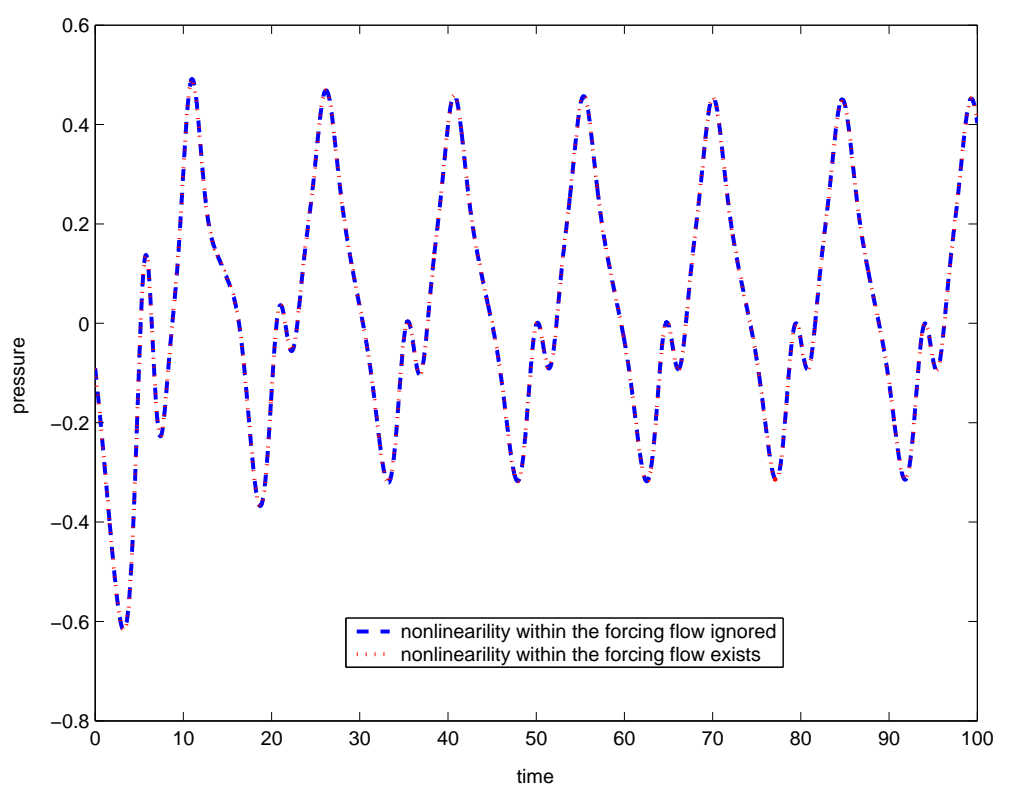

Figure 1. Output pressure at Mach 0.38 with and without nonlinearities in the forcing flow.

$\Gamma_{c}$ can be approximated by a constant, which has been normalized to unity. We can further rewrite Eq. (1) as Eq. (4), considering $V(t)$ as a scalar control input, in the form

$$
\dot{a}(t)=\bar{F}+\bar{G} a(t)+\left[\begin{array}{c}
a^{T}(t) \bar{H}^{1} a(t) \\
\vdots \\
a^{T}(t) \bar{H}^{N} a(t)
\end{array}\right]+\bar{P} V(t)+\left[\begin{array}{c}
\left(\bar{Q}^{1} V(t)\right)^{T} a(t) \\
\vdots \\
\left(\bar{Q}^{N} V(t)\right)^{T} a(t)
\end{array}\right],
$$

where $N$ is the number of modes, and the coefficient matrices of the system are defined by

$$
\bar{F}=\left[\begin{array}{c}
\bar{b}^{1} \\
\bar{b}^{2} \\
\vdots \\
\bar{b}^{N}
\end{array}\right], \quad \bar{G}=\left[\begin{array}{cccc}
\bar{d}^{11} & \bar{d}^{21} & \ldots & \bar{d}^{N 1} \\
\bar{d}^{12} & \bar{d}^{22} & \ldots & \bar{d}^{N 2} \\
\vdots & \vdots & \ddots & \vdots \\
\bar{d}^{1 N} & \bar{d}^{2 N} & \ldots & \bar{d}^{N N}
\end{array}\right], \quad \bar{H}^{k}=\left[\begin{array}{cccc}
\bar{g}^{11 k} & \bar{g}^{12 k} & \ldots & \bar{g}^{1 N k} \\
\bar{g}^{21 k} & \bar{g}^{22 k} & \ldots & \bar{g}^{2 N k} \\
\vdots & \vdots & \ddots & \vdots \\
\bar{g}^{N 1 k} & \bar{g}^{N 2 k} & \ldots & \bar{g}^{N N k}
\end{array}\right]
$$

and

$$
\bar{P}=\left[\begin{array}{c}
\bar{e}^{1} \\
\bar{e}^{2} \\
\vdots \\
\bar{e}^{N}
\end{array}\right], \quad \bar{Q}^{k}=\left[\begin{array}{c}
\bar{f}^{1 k} \\
\bar{f}^{2 k} \\
\vdots \\
\bar{f}^{N k}
\end{array}\right]
$$

The equation for the measured output is given by

$$
p(t)=M a(t),
$$

where $p(t)$ is the output pressure at the central cavity floor and $M$ is the coefficient matrix relating the pressure and the time coefficients using stochastic estimation. ${ }^{21}$

\section{Static Output Feedback Control}

In this section, an equilibrium analysis is first applied to the nonlinear POD based model described by Eq. (4). The equilibrium points of the nonlinear system are calculated and then the system is linearized 


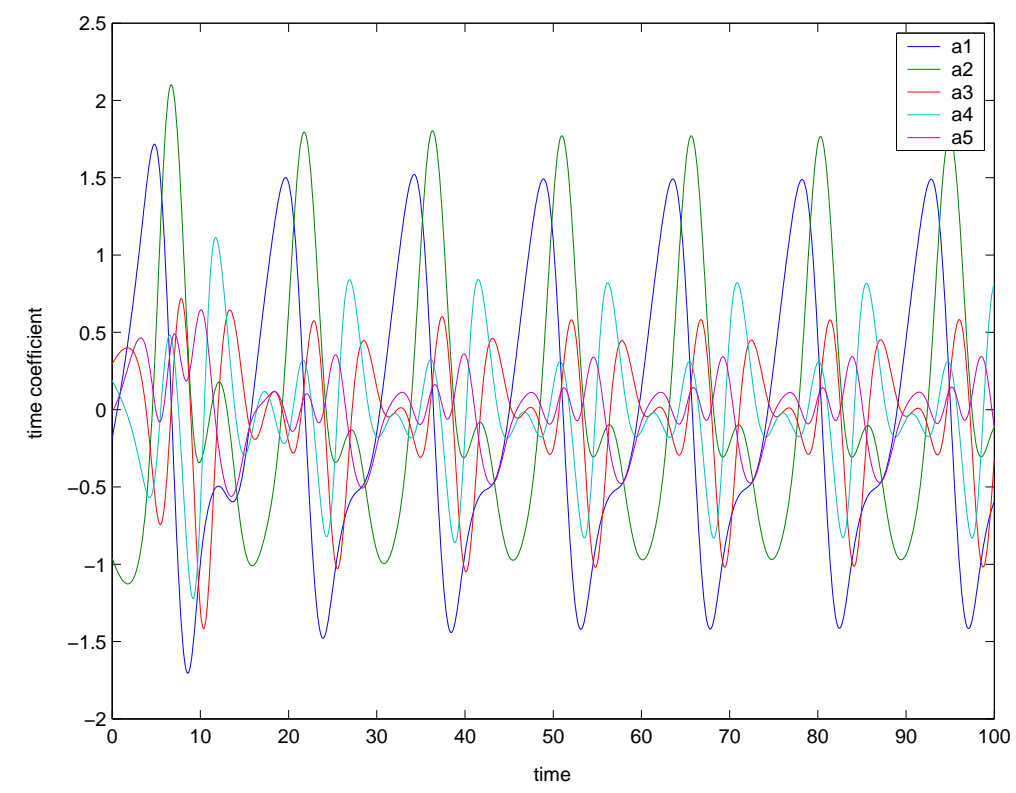

Figure 2. Time coefficients calculated by numerical simulation of the cavity flow at Mach 0.38: baseline

around the computed points. The effect of static output feedback control on the location of the equilibria is analyzed and discussed. It turns out that the control has very limited authority to modify the closed-loop equilibrium. Therefore, the POD model has been modified to shift the equilibrium to the origin of the state space, which corresponds to singling out the mean flow. The analysis on the effect of static output feedback control is then repeated for the modified model. All the computations and analysis have been performed on the data obtained using large eddy simulation of the cavity flow at Mach 0.38. A total of three cases have been studied: the baseline case and forced cases with the forcing sinusoidal signals at the frequency of $500 \mathrm{~Hz}$ and $900 \mathrm{~Hz}$, respectively. For the sake of brevity, only the results of the baseline case are reported.

\section{A. Equilibrium Analysis}

In order to gain insight into the properties of the nonlinear POD model, we started with determining the location of the equilibrium points of the unforced POD model. Since this task involves solving a nonlinear algebraic matrix equation derived from Eq. (4) with $V=0$, an analytic solution is intractable, and numerical methods must be sought. In our case, a Newton iterative method was implemented to calculate the equilibrium points of Eq. (4), that is, the roots of the nonlinear equation $f(a)=0$, where $f(a)$ is the right hand side of Eq. (4). The method implements the steepest descent iteration

$$
a_{k+1}=a_{k}-J^{-1}\left(a_{k}\right) f\left(a_{k}\right),
$$

where $J\left(a_{k}\right)$ is the Jacobian matrix

$$
J=\frac{\partial f(a)}{\partial a} .
$$

It should be noticed that the solution of the Newton method of Eq. (6) depends on the initial conditions and it is usually not unique. Evaluation of the time coefficients by numerical simulation shows that there exists a feasible region for the time coefficients given by

$$
\begin{aligned}
& \left|a_{1}\right|<1.72 \\
& \left|a_{2}\right|<2.11 \\
& \left|a_{3}\right|<1.42 \\
& \left|a_{4}\right|<1.23 \\
& \left|a_{5}\right|<0.65
\end{aligned}
$$

$$
4 \text { of } 11
$$


and so solutions obtained by means of the Newton algorithm that lie outside the feasible region need to be discarded. An example of the time history of the coefficients $a_{i}(t)$ of the POD model (4), obtained by means of computer simulations, is shown in Figure 2. The equilibrium solution $a_{0}$ relative to the baseline cavity flow at Mach 0.38 is computed as

$$
a_{0}=\left[\begin{array}{c}
0.1457 \\
0.1541 \\
0.0064 \\
0.0026 \\
-0.1370
\end{array}\right]
$$

The POD model Eq. (4) is linearized around the equilibrium $a_{0}$ to obtain

$$
\dot{a}=\left.\frac{\partial f(a)}{\partial a}\right|_{a=a_{0}} a:=J\left(a_{0}\right) a
$$

where the Jacobian matrix $J\left(a_{0}\right)$ is given by

$$
J\left(a_{0}\right)=\left[\begin{array}{ccccc}
0.0739 & -0.5392 & -0.1266 & -0.0188 & 0.0627 \\
0.4714 & 0.0525 & -0.3956 & 0.0808 & 0.0597 \\
0.0486 & 0.0545 & -0.1943 & 1.0659 & 0.2010 \\
0.0617 & 0.0221 & -0.7684 & -0.1731 & 0.0075 \\
-0.0451 & -0.0555 & -0.0730 & 0.0022 & -0.2352
\end{array}\right]
$$

The set of eigenvalues of Jacobian matrix is

$$
\lambda\left(J\left(a_{0}\right)\right)=\left[\begin{array}{c}
-0.1626+0.9363 i \\
-0.1626-0.9363 i \\
0.0401+0.4834 i \\
0.0401-0.4834 i \\
-0.2312
\end{array}\right]
$$

Note the presence of two unstable eigenvalues in $\lambda\left(J\left(a_{0}\right)\right)$. Very similar results have been obtained for the forced cases with forcing frequencies at $500 \mathrm{~Hz}$ and $900 \mathrm{~Hz}$.

\section{B. Static Output Feedback Control}

Static output feedback control, i.e, a control of the form $V=K p(t)$, is the simplest feedback law that can be implemented. To investigate the performance that can be obtained by static output feedback for different values of the gain $K$, we provide the plot of $K$ vs. the equilibrium point and the corresponding root locus, which shows how the location of the closed-loop eigenvalues change with respect to $K$. The first plot is reported in Figure 3, while the root locus is shown in Figure 4. Note that for $K \in[-\infty, 1058] \cup[2417, \infty]$, the equilibria of the closed-loop system change continuously and the eigenvalues change continuously as well. On the contrary, the Newton algorithm fails to yield a converging solution within the feasible region for $K \in[1059,2416]$.

Since very high gain solutions are of no practical use due to unavoidable limitations in the control authority, we restricted the analysis to a reasonable range of the feedback gain $K$. As shown in Figure 3, the sensitivity of the equilibrium solution is very small for $K \in[-100,100]$. Moreover, it is clear from Figure 4 that stabilization can not be achieved using proportional feedback. The forced cases show no significant difference from the baseline case, and similar conclusions can be drawn.

Since the presence of feedback control does not alter the location of the equilibrium, it makes sense to shift the coordinates of the state space to the unique equilibrium point $a_{0}$. Shifting the equilibrium of Eq. (4) to the origin corresponds to singling out the effect of the mean flow from the low order model, and considering the local behavior of the system around the mean flow. Letting

$$
\tilde{a}=a-a_{0}
$$




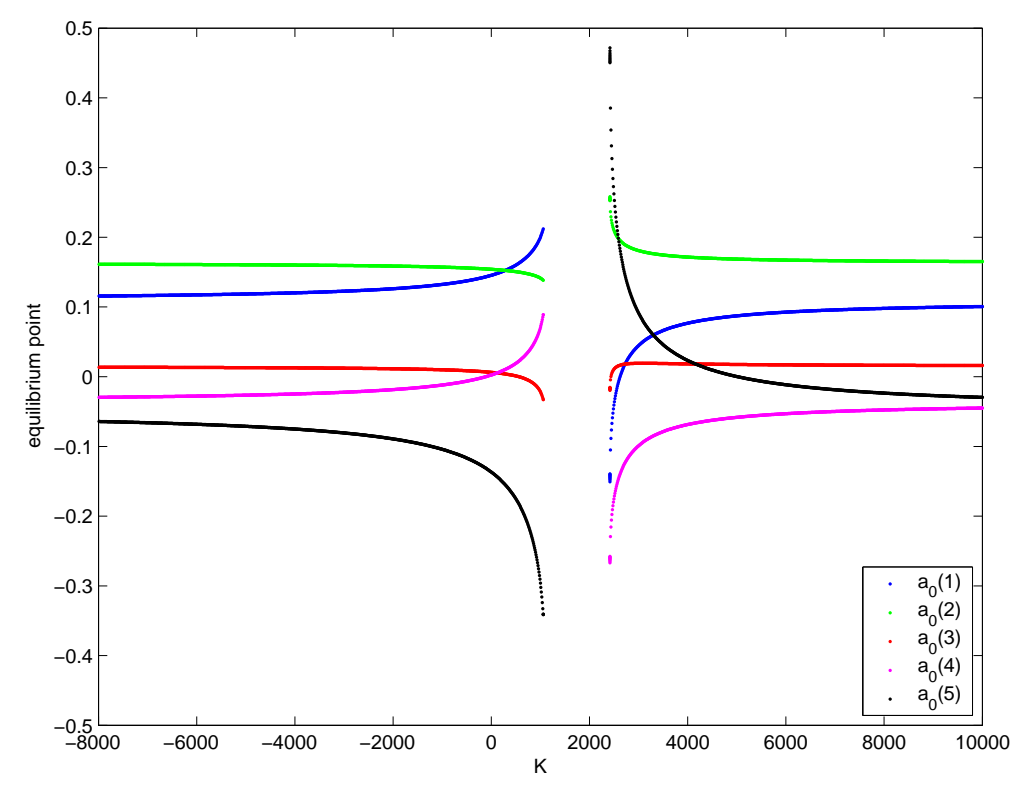

Figure 3. Equilibrium points vs. $K \in[-\infty, 1058] \cup[2417, \infty]$

we obtain

$$
\dot{\tilde{a}}=\bar{F}+\bar{G}\left(\tilde{a}+a_{0}\right)+\left[\begin{array}{c}
\left(\tilde{a}+a_{0}\right)^{T} \bar{H}^{1}\left(\tilde{a}+a_{0}\right) \\
\vdots \\
\left(\tilde{a}+a_{0}\right)^{T} \bar{H}^{N}\left(\tilde{a}+a_{0}\right)
\end{array}\right]+\bar{P} V+\left[\begin{array}{c}
\left(\bar{Q}^{1} V\right)^{T}\left(\tilde{a}+a_{0}\right) \\
\vdots \\
\left(\bar{Q}^{N} V\right)^{T}\left(\tilde{a}+a_{0}\right)
\end{array}\right]
$$

Recalling that $a_{0}$ is the equilibrium solution of the unforced system, that is,

$$
\bar{F}+\bar{G} a_{0}+\left[\begin{array}{c}
a_{0}^{T} \bar{H}^{1} a_{0} \\
\vdots \\
a_{0}^{T} \bar{H}^{N} a_{0}
\end{array}\right]=0
$$

the model in the new set of coordinates $\tilde{a}$ reads as

$$
\dot{\tilde{a}}=\widetilde{G} \tilde{a}+\left[\begin{array}{c}
\tilde{a}^{T} \widetilde{H}^{1} \tilde{a} \\
\vdots \\
\tilde{a}^{T} \widetilde{H}^{N} \tilde{a}
\end{array}\right]+\widetilde{P} V+\left[\begin{array}{c}
\left(\widetilde{Q}^{1} V\right)^{T} \tilde{a} \\
\vdots \\
\left(\widetilde{Q}^{N} V\right)^{T} \tilde{a}
\end{array}\right]
$$

Similarly, letting $\tilde{p}=p-p_{0}$, the output equation (5) can be rewritten as

$$
\tilde{p}=p-p_{0}=M a-M a_{0}=M \tilde{a} .
$$

The new modified model has the same structure as (4) and (5) but with the equilibrium transformed to the origin, which is more convenient for controller design and stability analysis.

The static output feedback $V=-K \tilde{p}$, which is the most appropriate for the model (16)-(17), does not alter the equilibrium at $a_{0}$ for the original POD model (4) even in presence of high-gain feedback, as opposite to the feedback law $V=-K p$ considered previously. The analysis reveals that the origin of (16) cannot be stabilized by the static output feedback $V=-K \tilde{p}$, as the root locus of the compensated system (shown in Figure 5) possesses two branches which remain inside the open right-half complex plane for all values of the gain $K$. It is quite obvious that static output feedback controllers are inadequate for the POD model (16)-(17), and that the use of state feedback or dynamic output feedback controllers is required.

6 of 11 


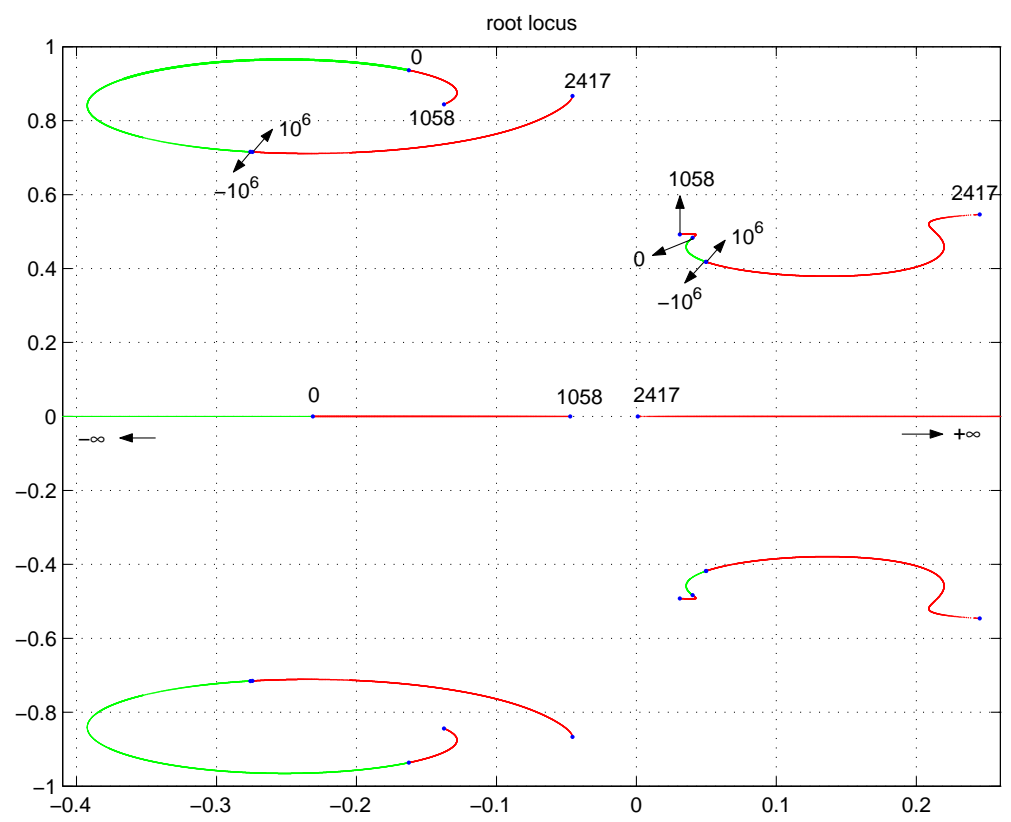

Figure 4. Root locus for $K \in[-\infty, 1058] \cup[2417, \infty]$

\section{Optimal State Feedback Control}

The jacobian linearization of (16) and (17) at the origin is readily obtained as

$$
\left\{\begin{array}{l}
\dot{\tilde{a}}=\widetilde{G} \tilde{a}+\widetilde{P} V \\
\tilde{p}=M \tilde{a}
\end{array}\right.
$$

It has been verified that the system (18) is controllable and observable for all considered cases. This allows standard state-space methodologies to be implemented to develop a controller (either by full state feedback or dynamic output feedback) to stabilize the origin of (18). This, in turn, yields a controller that locally stabilizes the origin of the nonlinear system (16). A convenient and well-established methodology for the controller design is offered by linear-quadratic optimal control. Let the cost function $J_{c}$ be

$$
J_{c}=\int_{0}^{\infty}\left(\tilde{a}^{T} Q_{w} \tilde{a}+V^{T} R_{w} V\right) d t
$$

where $Q_{w}>0$ and $R_{w}>0$ are the positive definite state weighting matrix and the scalar control weight, respectively. In our design, the weights have been chosen as $Q_{w}=I_{5 \times 5}$ and $R_{w}=1$. The solution of optimal state feedback controllers $V=-K_{i} \tilde{a}$, where the subscript $i=0,1,2$ stands for the baseline case, forced case with $f_{c}=500 \mathrm{~Hz}$ and $f_{c}=900 \mathrm{~Hz}$ respectively, have been obtained from the solution of the associated Riccati equations, and read as

$$
\begin{aligned}
K_{0}=\left[\begin{array}{lllll}
-1.6888 & -0.4917 & -0.0314 & 1.0509 & -0.2101
\end{array}\right]^{T} \times 10^{3} & \text { (baseline) } \\
K_{1}=\left[\begin{array}{lllll}
16.8 & -188.3 & 85.6 & 83.8 & 50.8
\end{array}\right]^{T} & \left(f_{c}=500 \mathrm{~Hz}\right) \\
K_{2}=\left[\begin{array}{lllll}
12.9 & -141.2 & 64.2 & 56.9 & 43.5
\end{array}\right]^{T} & \left(f_{c}=900 \mathrm{~Hz}\right)
\end{aligned}
$$

Similarly we design a full state observer in the form given by Eq. (21)

$$
\begin{aligned}
& \dot{\hat{a}}=\tilde{G} \hat{a}+\tilde{P} V+L(\tilde{p}-\hat{p}) \\
& \hat{p}=M \hat{a}
\end{aligned}
$$




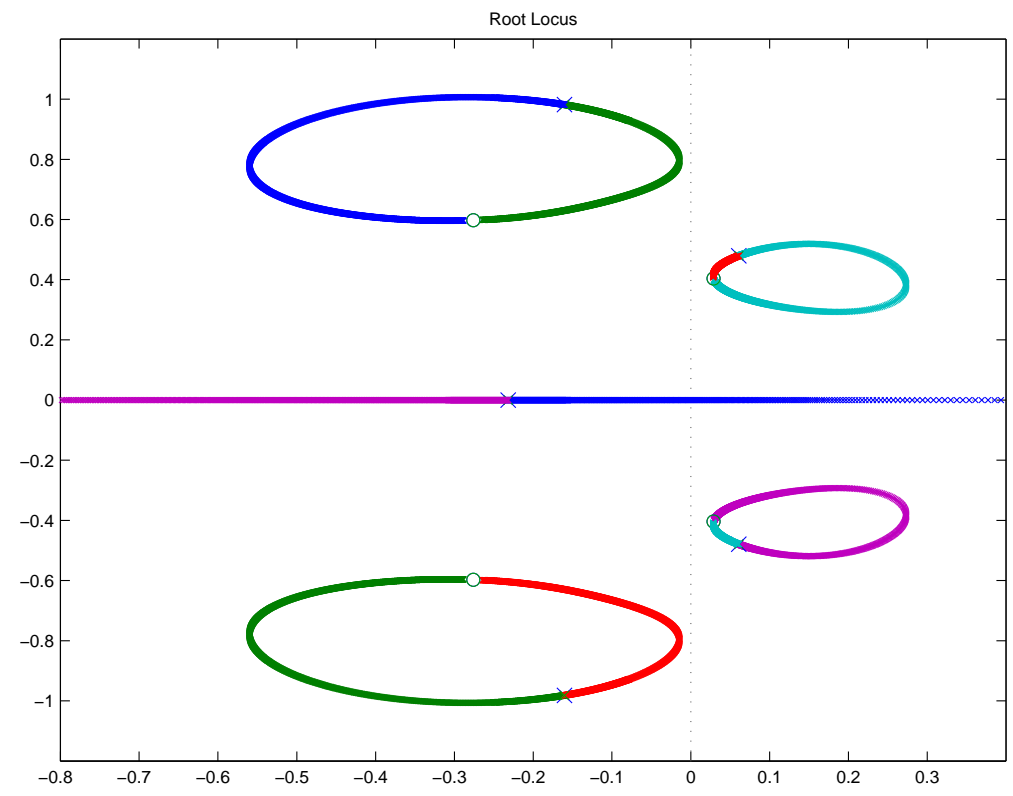

Figure 5. Root locus for the modified model with equilibrium at the origin.

where $\hat{a}, \hat{p}$ are respectively the estimated state and estimated output, and $L$ is optimal output-injection gain obtained by minimizing the cost function of the same form for the dual system. Choosing again the weights as identity matrices, the optimal $L_{i}$ for each case are given as follows,

$$
\begin{aligned}
& L_{0}=\left[\begin{array}{lllll}
-1.3 & 1.3 & -0.8 & -0.2 & -0.6
\end{array}\right]^{T} \quad(\text { baseline }) \\
& L_{1}=\left[\begin{array}{lllll}
-1.3 & 1.6 & -0.8 & -0.4 & -0.9
\end{array}\right]^{T} \quad\left(f_{c}=500 \mathrm{~Hz}\right) \\
& L_{2}=\left[\begin{array}{lllll}
-1.2 & 1.3 & -0.7 & -0.2 & -0.7
\end{array}\right]^{T} \quad\left(f_{c}=900 \mathrm{~Hz}\right) .
\end{aligned}
$$

\section{Simulation Results}

In this section, we show simulation results of the application of the optimal state feedback controllers and observer designed in Section IV to the nonlinear model described by Eq. (14). For all the three cases the robustness of the controllers to parameter variations has been evaluated. Since the controllers have been designed on the basis of the linearized models, their validity for the nonlinear model may be restricted to a possibly small neighborhood of the equilibrium. For this reason, estimates of the regions of attraction of each case have been obtained numerically.

\section{A. Controller Robustness}

Each controller given by Eq. (21) was designed for a fixed model, while it may be required to work under uncertain conditions, under the presence of disturbances, or even for a model different from the nominal one that the controller was designed for. Therefore, it is important to investigate the controller robustness. A simple test is to check if a controller designed on a specific case works for the other two cases as well. Denote the POD models obtained for the baseline case, the forced case with $f_{c}=500 \mathrm{~Hz}$ and $f_{c}=900 \mathrm{~Hz}$ by $P_{0}, P_{1}$, $P_{2}$ and the controller designed for each model by $C_{0}, C_{1}, C_{2}$, respectively. The result are given in Table 1 , where the entry 'yes' means that the controller can stabilize the corresponding linear model and the entry 'no' means that the controller can not stabilize that model. 
Table 1. Robustness of the optimal LQ controllers

\begin{tabular}{c|ccc} 
& $C_{0}$ & $C_{1}$ & $C_{2}$ \\
\hline$P_{0}$ & yes & no & no \\
$P_{1}$ & no & yes & yes \\
$P_{2}$ & no & yes & yes
\end{tabular}

\section{B. Region of Attraction}

An important aspect of the design is to evaluate the region of attraction of the origin in closed loop provided by each controller. As a matter of fact, since the design is based on linear control theory for the linearized model, attractivity is guaranteed only for initial conditions within a (possibly small) neighborhood of the origin of the nonlinear model. For the nonlinear system (16), a theoretical analysis of the region of attraction is difficult and beyond the scope of the present paper. Instead, the region of attraction obtained by each controller $K_{i}$ can be estimated numerically. For the sake of simplicity, we will discuss the estimation of the region of attraction for the system in closed-loop with the state-feedback controller $V=-K_{i} \tilde{a}$ only. Since the region of attraction is the largest open invariant set which has the property that each point of the set is asymptotically attracted to the origin, an estimate of the domain of attraction is obtained looking for compact sets which contain the origin in their interior, are invariant under the flow of the closed-loop system, and such that all the point at its boundary are attracted to the origin.

The obvious choice for the compact sets in question is given by the level sets of the Lyapunov functions for each linearized closed-loop system, that is the functions

$$
\mathcal{V}^{i}(\tilde{a})=\tilde{a}^{T} S^{i} \tilde{a}, \quad 0 \leq i \leq 2,
$$

where $S^{i}$ is the solution of the Lyapunov equation

$$
S^{i}\left(\tilde{G}-\tilde{P} K_{i}\right)+\left(\tilde{G}-\tilde{P} K_{i}\right)^{T} S^{i}=-I
$$

corresponding to the given choice for $K_{i}, 1 \leq 0 \leq 2$. The level sets $\Omega_{c}^{i}$ are defined as

$$
\Omega_{c}^{i}=\left\{\tilde{a} \in \mathbb{R}^{N} \text { such that } \mathcal{V}^{i}(\tilde{a}) \leq c\right\}
$$

where $c$ is a positive constant. The boundary of $\Omega_{c}^{i}$, denoted by $\partial \Omega_{c}^{i}$ is a closed $N-1$-dimensional surface in $\mathbb{R}^{N}$. Fix $c>0$, and consider, for the system (16) in closed-loop with the state feedback controller $V=-K_{i} \tilde{a}$, arbitrary initial conditions $\tilde{a}(0)$ such that $\tilde{a}(0) \in \partial \Omega_{c}^{i}$. Integrating numerically the differential equations of the closed-loop system allows to determine whether initial conditions in $\partial \Omega_{c}^{i}$ are attracted to the origin or not. The search is initialized with $c>0$ small enough so that the state trajectory with the corresponding initial condition converges to the origin. We enlarge $c$ until we find $c_{\max }$ such that the state trajectory with initial condition $\partial \Omega_{c}^{i}$ diverges for any $c>c_{\max }$. Then, an estimate of the region of attraction is given by the corresponding level set $\Omega_{c_{\max }}^{i}$. Similarly to the analysis of the controller robustness, we apply a linearly designed controller to the nonlinear model described by Eq. (16) for all three cases. The estimated region of attraction represented by the value of $c_{\max }$ is given in Table 2 , where a value $c_{\max }=0$ corresponds to an unstable equilibrium at the origin in closed-loop, consistently with the results given in Table 1. Finally,

Table 2. Region of attraction of the optimal LQR state-feedback controllers

\begin{tabular}{c|ccc} 
& $C_{0}$ & $C_{1}$ & $C_{2}$ \\
\hline$P_{0}$ & 4 & 0 & 0 \\
$P_{1}$ & 0 & 0.9 & 1.1 \\
$P_{2}$ & 0 & 7 & 11
\end{tabular}

an example of simulation results is given in Figure 6 and Figure 7, which show respectively the output $\tilde{p}(t)$ and the first time coefficient $\tilde{a}_{1}(t)$ for each the closed-loop systems $\left(P_{0}-C_{0}\right),\left(P_{1}-C_{1}\right)$, and $\left(P_{2}-C_{2}\right)$ corresponding to the main diagonal in Table 1 and Table 2. For each case, the initial condition has been selected within the estimated region of attraction, and the corresponding trajectory decays asymptotically to the origin. 

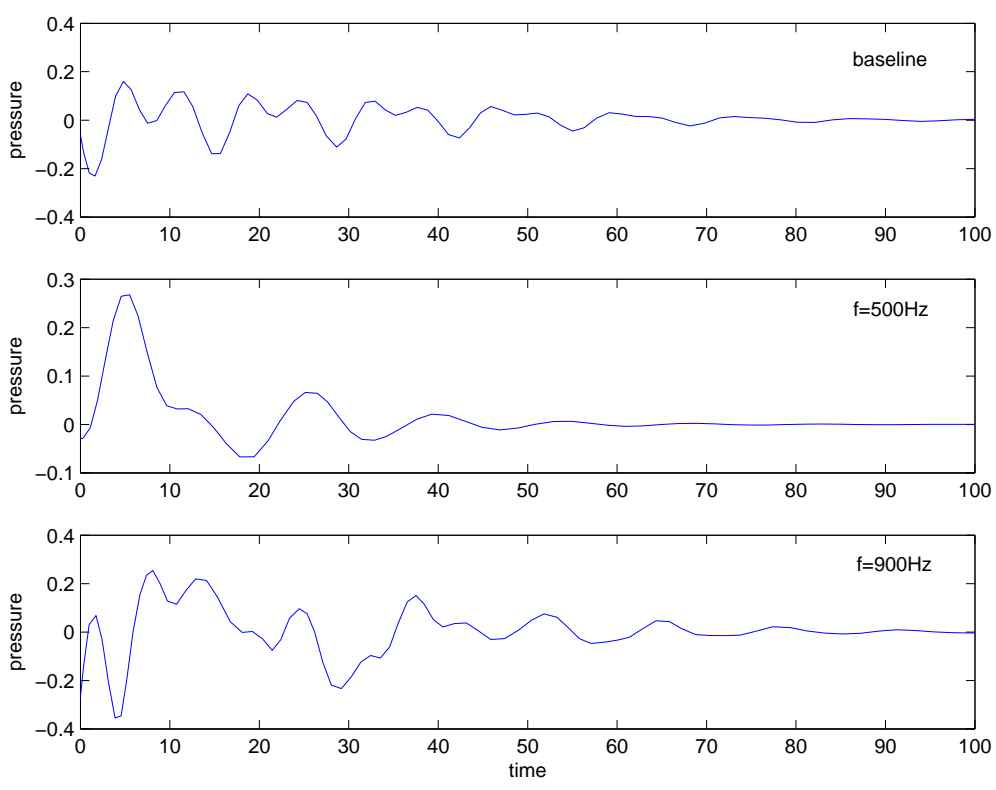

Figure 6. Simulation results - Output pressure $\tilde{p}(t)$.

\section{Conclusion}

In this paper, we have reported preliminary studies on POD based low dimensional modeling and feedback controller design for the control of cavity flows. It has been shown that the equilibrium of the nonlinear POD model corresponding to the mean flow is exponentially unstable and cannot be stabilized using a static output feedback controller. A modified model with the equilibrium transformed to the origin has been derived and further analyzed. A Linear Quadratic (LQ) optimal state feedback controller and an LQ optimal observer has been developed for the linearized models, and its effectiveness tested on the nonlinear POD model. Numerical analysis has been used to test the controller robustness and give an estimate of the region of attraction. We are currently investigating real time implementations of the proposed controllers in our experimental setup. ${ }^{2}$ An extension of this work will consider the design of nonlinear feedback controller on the basis of the analytical nonlinear POD models to achieve better closed-loop performance in terms of transient response, robustness and size of the domain of attraction.

\section{Acknowledgments}

This work is supported in part by AFRL/VA and AFOSR through the Collaborative Center of Control Science (Contract F33615-01-2-3154), and by DAGSI. Computer simulation work is supported in part under NASA Glenn's Aerospace Propulsion and Power Base Research and Technology Program and in part by a grant of computer time from the DOD High Performance Computing Modernization Program at the Army Research Laboratory. We would like to thank Marco Debiasi and James Malone for their help.

\section{References}

${ }^{1}$ Samimy, M., Debiasi, M., Caraballo, E., Özbay, H., Efe, M. Ö., Yuan, X., DeBonis, J., and Myatt, J. H., "Closed-Loop Active Flow Control - A Collaborative Approach," AIAA paper 2003-0058, Jan. 2003.

${ }^{2}$ Samimy, M., Debiasi, M., Caraballo, E., Malone, J., Little, J., Özbay, H., Efe, M. Ö., Yan, P., Yuan, X., DeBonis, J., Myatt, J. H., and Camphouse, R. C., "Exploring Strategies for Closed-Loop Cavity Flow Control," AIAA paper 2004-0576, Jan. 2004.

${ }^{3}$ Caraballo, E., Samimy, M., and DeBonis, J., "Low Dimensional Modeling of Flow for Closed-Loop Flow Control," AIAA paper 2003-0059, Jan. 2003.

${ }^{4}$ Yuan, X., Efe, M. Ö., and Özbay, H., "On Delay-Based Linear Models and Robust Control of Cavity Flows," Advances in Time-Delay Systems, edited by S. Niculescu and K. Gu, Vol. 38, Springer-Verlag, 2004, pp. 287-298. 

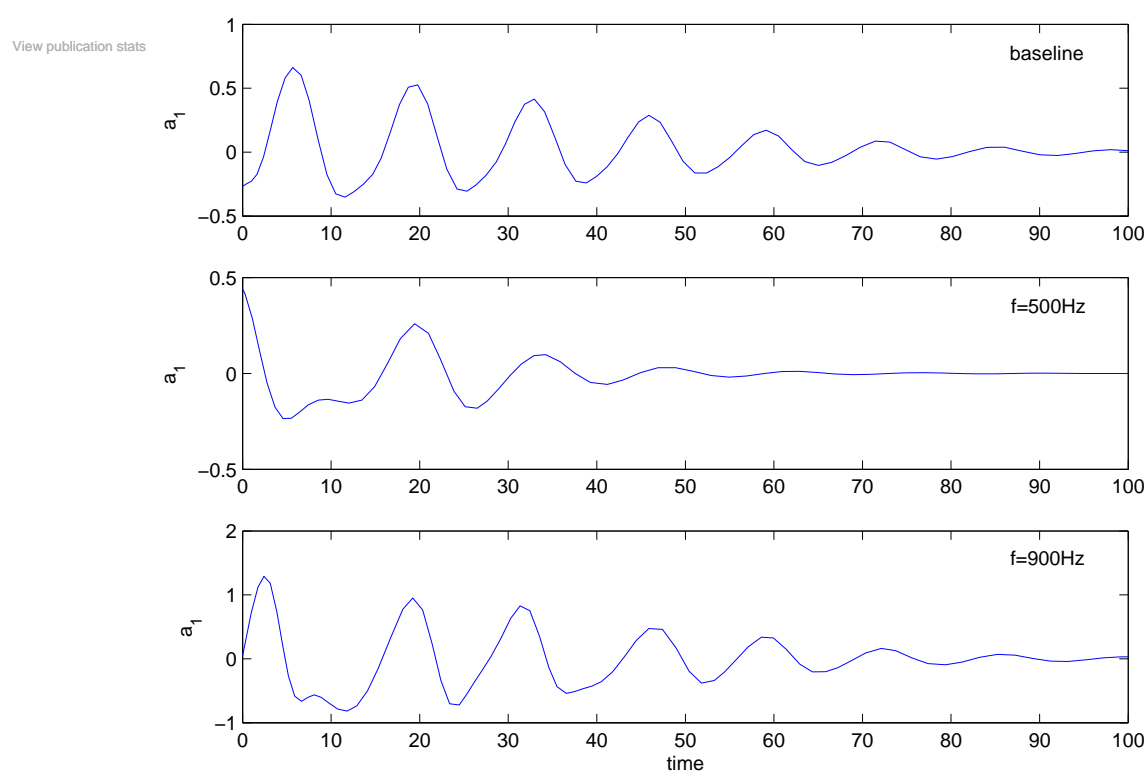

Figure 7. Simulation results - Time coefficient $a_{1}(t)$.

${ }^{5}$ Yan, P., Debiasi, M., Yuan, X., Caraballo, E., Efe, M. Ö., Özbay, H., Samimy, M., DeBonis, J., Camphouse, R. C., Myatt, J. H., Serrani, A., and Malone, J., "Controller Design for Closed- Loop Control of Cavity Flows," AIAA paper 2004-0573, Jan. 2004.

${ }^{6}$ Debiasi, M. and Samimy, M., "Logic-Based Active Control of Subsonic Cavity-Flow Resonance," AIAA Journal, Vol. 42, No. 9, 2004, pp. 1901-1909.

${ }^{7}$ Debiasi, M. and Samimy, M., "An Experimental Study of the Cavity Flow for Close-Loop Flow Control," AIAA paper 2003-4003, June 2003.

${ }^{8}$ Colonius, T., "An Review of Simulation, Modelling and Active Control of Flow/Acoustic Resonance in Open Cavities," AIAA paper 2001-0076, Jan. 2001.

${ }^{9}$ Cattafesta, L. N., III, Williams, D. R., Rowley, C. W., and Alvi, F. S., "Review of Active Control of Flow-Induced Cavity Resonance," AIAA paper 2003-3567, June 2003.

${ }^{10}$ Cattafesta, L. N., III, Garg, S., Choudhari, M., and Li, F., "Active Control of Flow-Induced Cavity Response," AIAA paper 1997-1804, June 1997.

${ }^{11}$ Rowley, C. W., Williams, D. R., Colonius, T., Murray, R. M., MacMartin, D. G., and Fabris, D., "Model-Based Control of Cavity Oscillations Part II: System Identification and Analysis," AIAA paper 2002-0972, Jan. 2002.

${ }^{12}$ Rowley, C. W. and Williams, D. R., "Control of Forced and Self-Sustained Oscillations in the Flow Past a Cavity," AIAA paper 2003-0008, Jan. 2003.

${ }^{13}$ Williams, D. R., Rowley, C., Colonius, T., Murray, R., MacMartin, D., Fabris, D., and Albertson, J., "Model-Based Control of Cavity Oscillations Part I: Experiments," AIAA paper 2002-0971, Jan. 2002.

${ }^{14}$ Cabell, R. H., Kegerise, M. A., Cox, D. E., and Gibbs, G. P., "Experimental Feedback Control of Flow Induced Cavity Tones," AIAA paper 2002-2497, June 2002.

${ }^{15}$ Caraballo, E., Samimy, M., Narayanan, S., DeBonis, J., and Scott, J., "Application of Proper Orthogonal Decomposition to a High Speed Axisymmetric Jet," AIAA paper 2001-2783, June 2001.

${ }^{16}$ Citriniti, J. and George, W., "Reconstruction of the Global Velocity Field in the Axisymmetric Mixing Layer Utilizing the Proper Orthogonal Decomposition," Journal of Fluid Mechanics, Vol. 418, 2000.

${ }^{17}$ Cohen, K., Siegel, S., and McLaughlin, T., "Proper Orthogonal Decomposition Modeling of a Controlled Ginzburg-Landau Cylinder Wake Model," AIAA paper 2003-1292, Jan. 2003.

${ }^{18}$ Rowley, C. W., Colonius, T., and Murray, M., "Dynamical Models for Control of Cavity Oscillations," AIAA paper 2001-2126, May 2001.

${ }^{19}$ Samimy, M., Debiasi, M., Caraballo, E., Özbay, H., Efe, M. Ö., Yuan, X., DeBonis, J., and Myatt, J. H., "Development of Closed-Loop Control for Cavity Flows," AIAA paper 2003-4258, June 2003.

${ }^{20} \mathrm{Efe}, \mathrm{M}$. Ö. and Özbay, H., "Low Dimensional Modelling and Dirichlet Boundary Controller Design for Burgers Equation," International Journal of Control, Vol. 77, No. 10, 2004, pp. 895-906.

${ }^{21}$ Adrian, R. J., "On the Role of Conditional Averages in Turbulent Theory," Turbulence in Liquids, Science Press, Princeton, 1977. 